\title{
Getting the message about biobanking: returning to the basics
}

\section{Daniel R Catchpoole}

The Tumour Bank, The Children's Cancer Research Unit, The Kids Research Institute, The Children's Hospital at Westmead, Westmead, NSW, Australia
Correspondence: Daniel R Catchpoole The Tumour Bank, Children's Cancer Research Unit, The Kids Research Institute, The Children's Hospital at Westmead, Locked Bag 400I, Westmead, NSW, 2145 , Australia

Tel +6I 29845 I205

Fax +6I 298453078

Email daniel.catchpoole@health.nsw.gov.au
This article was published in the following Dove Press journal: Journal of Biorepository Science for Applied Medicine 16 January 2017

Number of times this article has been viewed
Abstract: Much has been written about biobanks and biobanking. Since biobanking rose to prominence in the 1990s and became a scientific discipline in its own right, the collection and distribution of human tissue samples or "biospecimens" has been the subject of much critique, debate, and assessment. However, what is the message these discussions provide the wider research community? Are they reflective of progression of a new discipline? Do they represent clarity in the field or confusion? At one point we are told that biobanks are vital infrastructure, yet in the next breath we are told that biobanks are complex, full of risk, inefficient, and unsustainable. Biobanks struggle to receive sustainable funding with many restructuring or closing down, producing dilemmas about what to do with the legacy resources. This review critiques five key messages being relayed by the biobanking community, identifying the five messages that are actually being received by the research community. It also presents a "back to basics" view for biobanking that will return our attention back to five fundamental principles that should guide the ongoing discussion. This includes that biobanking is about the active provision of tissue for research that is vital for improved health outcomes. This activity is founded in human interrelationships, is already performed routinely within hospitals, and is driven by the opportunity to learn from the information contained within the biospecimens. The examples provided will be drawn from New South Wales, Australia, where building a state-wide biobanking infrastructure has been implemented, and which highlight areas where back to basics approach will be beneficial. A back to basics view of biobanking will highlight that the current positions proposed to deal with these issues are mere "straw men" solutions and that the need for a fundamental shift in our thinking is the real issue that needs to be addressed.

Keywords: fundamentals, translational research, exceptionalism, bio-objectification, ethics, information

\section{Introduction}

Biobanks are vital research infrastructure that are absolutely fundamental for productive and meaningful translational research in human diseases like cancer.

Or so we are told!

The growth of the biobank industry over the past 20 years has been identified by Time magazine as one of the top 10 innovations in 2009. ${ }^{1}$ Indeed, the prevalence of literature detailing biobank structures, operations, practice, and purpose demonstrates how the discipline of biobanking has embedded itself into the mindset of biomedical researchers. Biobanks are found in almost every country, ${ }^{2,3}$ medical research institute and university, ${ }^{4,5}$ comprehensive cancer centres, ${ }^{6}$ and hospitals. ${ }^{7}$ Major research groups

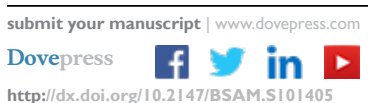


spend considerable time building biobanks to underpin their investigations, whereas multicenter research programs rely on biobanking networks to gather the tissue samples that they require. ${ }^{8}$ Biobanks can be generic "poly banks," collect samples from broad disease types ${ }^{4,10}$ or rare diseases,,${ }^{1-13}$ store residual tissue from diagnostic procedures, ${ }^{14}$ focus on pediatrics, ${ }^{15}$ or support specific research themes such as drug discovery ${ }^{16}$ and post-genomic era. ${ }^{17}$

As a result of increased biobanking activities, there has been an exponential growth of diverse opinions related to biobank operations, purpose, and placement within the translational research landscape. Biobank activity includes many other biomedical research disciplines, inviting a range of "expert" commentary on what should be done and how they should function. Biobanking has been considered, on the one hand, "glorified pathology specimen collection" (anonymous comment, Royal College of Pathologist Australasia Pathology Update Conference, Melbourne, Australia, 2010) and, on the other hand, an "impressive scientific and technological development that ... is the cornerstone of the current edifice of biomarker research."16 As we overlay such commentary with further discussion regarding purpose, effectiveness, efficiency, risk, control, regulation, governance, management, certification, and standardization of biobanks, we find ourselves burgeoning with detailed viewpoints that have become a din such that it becomes difficult to discern what is meaningful. This review highlights how the messages that biobanks attempt to provide lead to a more confused undertaking for biobankers. It presents how many of the issues that are currently being faced would be solved with a refocus on more foundational practices related to the use of human tissue in translational research. It also discusses five key messages that underpin the general views about biobanking for human diseases as presented by the biobanking community and then identifies the emergent messages being heard by the wider research community that have led to the complex, conflicted, and often confused state of biobanking we have today. It then discusses five key fundamental principles that will provide clarity in our thinking and simplify our biobanking practice. Examples used in this review represent the author's experience in Australia and, specifically, the state of New South Wales (NSW) but likely reflect situations experienced around the world.

\section{Biobanking messages: what we are told, what we hear Message I: Biobanks are vital for medical research}

Tissue banks represent essential resources and platforms for biomedical research serving basic, translational and clinical research projects by providing human tissue and their derivatives as well as offering access to key technologies. ${ }^{18}$

Translational research in medicine mandates that tissue from patients be collected and used for research studies and that these biospecimens should be obtained from highly specialized biorepositories. ${ }^{14,15,19,20}$ It is considered that personalized medicine, as the future of clinical oncology, ${ }^{21}$ will only realize its potential once the research discovery and its clinical validation are facilitated through open access to biospecimens collected at key points during a patient's clinical journey. ${ }^{10,16,22}$

With the value of systematic collection of tissues within translational research emerging as a key element to its success, biobanks are now being relied upon to standardize tissue collection for improved science quality, statistical power provided by sample size, ensure public safety, and actually drive research. ${ }^{3}$ In Australia, a strategic review of biomedical research made recommendations that biobanks be established ${ }^{23}$ while at the same time, in NSW, a similar review recommended that a state-wide biobanking infrastructure be developed to underpin biomedical research. ${ }^{24}$ During this time, biobanks have moved from isolated, researcherinitiated collections into central elements of major research infrastructure.

With the emphasis on the "essential" nature of biobanks to research infrastructure, ${ }^{3,25}$ three key questions arise, which confounds our reasoning around biobanking:

1. What is a biobank? With a broad requirement for biobanking activity, the distinctive features that characterize biobanks need to be identified before workable frameworks around establishing biobanks can be formed. The Organisation for Economic Co-operation and Development defines biobank as "a collection of biological material and the associated data and information stored in an organised system, for a population of a large subset of a population." 12 In Australia, the term "biobank" is a general descriptor for any collection of biospecimens that may be used for research; ${ }^{26}$ however, there is no mention of "systematic collection" or professional standards as a distinctive. ${ }^{27}$ As policies are being drafted in NSW, biobanks are broadly defined in generic terms, as "an entity that receives, stores, processes and/or distributes specimens for approved research." 28 This has led to a situation where researchers applying to ethics committees to undertake the specific collection of biospecimens for their own limited research project are being prohibited from commencing their studies until they present a full range of standard operating procedures, establish biobank 
committees, draft ethically defensible plans (EDPs), and undergo a regulatory process in the same manner as major service provider biobanks.

2. What motivates us to build them? Hewitt and Watson ${ }^{29}$ state that currently biobanks are not defined on the basis of "purpose." In Australia, during 2004, a heightened period of direct governmental funding for biobanking, ${ }^{30}$ the absence of a clear definition of a biobank and its purpose led to the sudden expansion where any tissue collection became a biobank! However, this became a point of contention and failure when, in 2009, as soon as these funding schemes were wound back, some "biobanks" suddenly reverted to "cohort studies," "tissue resources," or "clinical collections" that could be funded as part of research studies rather than as specific research infrastructure. This highlights that when the motivation to "be a biobank" is right, collections will be "biobanks" as long as the motivation remains. How biobanks are defined will not only determine what a biobank is but also indicate their purpose. However, mere definitions do not indicate the motivations of those building the biobank infrastructure. Motivation can be captured in policy which sets boundaries of practice of the biobank and reflects the core values of the different authorities, community groups, and stakeholders who consider it a "biobank." 31

3. How distinctive are they from other tissue-handling activity? On the one hand, we are told that every tissue collection is a biobank. Zatloukai and Hainaut ${ }^{16}$ however make a distinction between biobanked tissues used in drug discovery research and those used in diagnostic or therapeutic applications. There is a propensity to distinguish biobanking from other activities including research using samples that have not been specifically stored for further use, repositories for human tissue that are created for diagnostic or clinical purposes, for-profit biobanks that have commercial interests, clinical trials, and specialist units such as Newborn Bloodspot Screening. ${ }^{32}$ Indeed, these are specifically excluded in the policy being drafted to guide the NSW state-wide infrastructure plan. ${ }^{28}$ This creates an unhelpful dichotomy. Here is the contradiction. All these examples are practices that "receive, store, process and distribute specimens," often for research and easily fit accepted definitions of biobanking. All these operations seek to transfer human tissue samples from a donor to an investigator, often to discover some new finding, most often to inform on the individual patient, and to meet the same criteria that define a biobank. How these activities are different from "biobanking" is not apparent.

Policy and practice documents are not sufficiently detailed to draw out any meaningful distinction that reflects a definition of a biobank. On the one hand, we have biobanks needing a specific definition and on the other hand, when defined, every tissue-handling enterprise can fall within the definition of a biobank. The significance of this dichotomy means that definitional issue around biobanking makes it difficult to establish the ethical boundaries. ${ }^{33}$ One of the major obstacles to develop a uniform system of regulation is the lack of an agreed definition of "biobank."3,34,35 By unwittingly creating this identity crisis, we are finally left with the question of whether biobanks are really vital for the translational research to occur or can tissues be obtained from some other facility?

Consequence of message 1: Biobanks have an identity crisis.

\section{Message 2: Biobanking is a specialist discipline}

Biobank activity is developing as a progressively complex young discipline playing a central role in biomedical research. ${ }^{36}$

Often, biobanking is presented as an emerging and specialist discipline, requiring the development of new standards and procedures. The systematic collection of human tissues for the purposes of research is presented as a novel strategy in biomedical research requiring new management structures and interdepartmental tissue-handling pathways. ${ }^{7}$ Recently, we have been told to collect the "next generation biospecimens." Our tissue collection procedures are to ensure that biopsied samples are representative, viable, and adequate for subsequent molecular applications, recognizing how we handle biospecimens may influence the results of subsequent tests. ${ }^{17}$ This is a classic attempt presenting that biobanking is novel, worthy of special attention and, moreover, requires specific funding. However, the goal of all tissue handling undertaken by research as well as clinical and pathology departments is to ensure that the tissue samples are handled in a manner that best represents the disease and is therefore fit-for-purpose.

The systematization of tissue collections is not a new phenomenon. Rudolf Virchow, the "father of modern day anatomical pathology," proposed systematic collection 
of biospecimens for the ongoing study and exploration of patient materials in mid/late-1800s. Dr Frantisek Kral established the first service collection of microorganisms in 1896. Systematic tissue collections were established by the US Navy which commenced biobanking of human specimens in the $1950 \mathrm{~s}^{37}$ and the USSF AIDS Specimen Bank in 1982. ${ }^{4}$ A US-based survey found a remarkable diversity in organizational structures across its national biobanks, typical of an industry that is rapidly fragmenting rather than emerging into a single discipline. ${ }^{38}$ In NSW, cancer biobanks were similarly organized in various ways for $\sim 20$ years, ${ }^{39,40}$ leading to the claim that it is a "cottage industry" rather than an emerging new, vital field. Hence, this specialized discipline is not a new activity but rather a practice that has a fresh prominence within the organizational structures that requires specialized management. This has led to two pervasive and conflicting considerations that keep biobanks as isolated entities within the organization and threaten their sustainability: exceptionalism and objectification.

1. Biobank exceptionalism is defined as "a view that biobank research is so special that it requires different moral and legal standards, either more stringent or more lax, often the latter." ${ }^{\prime 41}$ Biobanking exceptionalism produces dichotomies within our policy base, making rules that apply to few but not others although it does the same thing. For example, focusing on the practice of patients being "consented for biobanking" emphasizes that the collection and storage of tissue biospecimens in freezers is the primary goal of the activity and is an end in itself. It gives weight to the notion that a biobank is a new form of facility that is unique and original in its operations, practices untested processes that are inherently different from any other practice ongoing with biomedical research that has unknown consequences and impact. Furthermore, only biobanks are able to do this activity, consequently needing special scrutiny, different regulatory oversight, ethical clearance, and governance obligations. Biobank exceptionalism considers that biobanks are motivationally and operationally exclusive from other groups who handle human tissue specimens. This is a flawed principle that is leading to complexity within our policy frameworks.

2. Bio-objectification is a process that describes the sociotechnical-material relationship, where human tissue shifts from being considered "human" to being an object relevant to the research process that can become informative to clinico-researchers through scientific process. ${ }^{42-44}$ It determines how biospecimens are seen in the research continuum and causes the value of tissue resources to be contextualized on the basis of the dependence of the resource to the institutional relationships, internal and external, and academic and societal. ${ }^{45}$ When biobank momentariness is considered as a resource that is no longer deemed sustainable, bio-objectification allows an organization to ignore the human aspects of the biospecimens and consider them "waste" and discard them. ${ }^{44}$ Biobanking has brought a shift on how we value tissues and bio-objectification allows biospecimens to be seen as a commodity, an information source, a research "product" that may or may not be needed or an item that can be sold on the free market. ${ }^{46}$

Exceptionalism states that biobanks and biospecimens are special and that institutions should change to get the most out of them to give them purpose and value. Bio-objectification says that biobanks and biospecimens are valuable only within the context of what the institution/society want to achieve. If biobanks do not fit with the goals of the institution, they must change so that it becomes useful to them or they will have no purpose and the biospecimens can be discarded as "waste." Does the intrinsic value of the biospecimens necessitate that the institution or research community give them exclusive attention and set up specific sets of rules or policies? Or does the need of the biomedical research community to advance their understanding of disease place a specific value on the biospecimens and require the facilities to manage them as there is need? The net result is that health organizations attempt to manage biobanks as separated resources requiring unique considerations.

Consequence of message 2: Biobanks require unique attention.

\section{Message 3: Biobanking is responsible for the ethical usage of tissue}

\footnotetext{
Biobanks involved a delicate balance between health policy objectives, academic research, public good outcomes, community trust in the benefits of biobanks and protection of public interests such as privacy. ${ }^{12}$
}

We are told that as a new discipline biobanking requires heightened ethics committee scrutiny and governance oversight. ${ }^{47,48}$ As clinical annotation of biospecimens provides them their research value, distributing human tissues to researchers personalizes the donors within the research domain leading to biobanks taking on the stewardship responsibilities for the tissue..$^{33,49}$ As a consequence, 
biobanks are required to follow "good practice" to manage risk related to the use of tissues in research. ${ }^{50}$ Good outcomes in tissue handling are therefore driven by neither the exceptionality of the biobanking facility nor its vitality to biomedical research success, but rather through overcoming the "numerous methodological, ethical, legal and technical problems." $9,33,51,52$ Although biobanks attempt to limit the risk in the following ways, considerable controversy still remains.

1. Structuring ethics and consent standards: Systematic qualitative study of the opinions to cancer biobanking performed at public and private hospitals in NSW demonstrated that informed consent for general use of biospecimens for future unspecified research was given willingly by the majority of patients and parents of children and was supported by lay persons. ${ }^{53,54}$ Worldwide, reports regarding informed consent for the collection of tissues for future unspecified research by biobanks ${ }^{5,56}$ indicate that willingness to consent was independent of all factors including age, sex, and tumor type. Yet, debates about the consents, its scope (broad or specific), the merits of opt-in and opt-out consenting, and content of consent requests have not been resolved. ${ }^{14,55,57-61}$ In NSW, attempts to resolve these issues have led to the development of a common consent form as part of the state-wide biobanking plan. ${ }^{24}$

2. Understanding autonomy: Biobanks strive to respect patients by ensuring that informed consent has been obtained. Patient autonomy in the consent decisionmaking process is presumed paramount, ${ }^{41}$ even for children. ${ }^{62,63}$ Within biobanking, the consenting process is often reduced to a crude imperative to gain a signed form rather than a discussion about "empowerment," "engagement," and "education" of the donor in the research process. ${ }^{60}$ Hansson $^{61}$ states, "autonomy is inherently social." Here the inter-subjectivity of a patient's autonomy highlights that individual human beings can participate in the formulation of a moral code for all. ${ }^{62}$ Hence, while we strive to manage risk by ensuring patient empowerment over their decision to donate tissues through "informed consent," we also engage in public education about biobanking shaping the opinions of the community toward translational research. For example, it has been questioned whether children should be allowed to develop autonomy and to provide broad biobanking consent on their own behalf. ${ }^{63,64}$ Yet when patients are asked directly about this, equivocal responses are obtained, suggesting ambivalence or naivety of lack of knowledge of the significance of what they are being asked. ${ }^{65,66}$ It is noteworthy that with community education, there will be heightened scrutiny of our practices identifying new risks which will require wider vigilance.

3. Return of results and EDPs: The age of genomics and focus on "personalised medicine" ${ }^{97,68}$ have perpetuated the debate regarding whether clinically informative and actionable results discovered during the research process be returned to the donors. ${ }^{49,69}$ This concern reflects, among other things, the principle that biospecimens personalize research. ${ }^{50}$ However, if custodianship of biospecimens lies with the biobanks, then the biobanks would be responsible for the mechanisms that are used to assess the "clinical relevance" of research results and the level of intervention that can be expected. ${ }^{9}$ Yet, according to Australia's National Statement (Section $3.4),{ }^{70}$ this responsibility lies with the researchers using the biospecimens who must develop and seek approval of their EDP before they can proceed. An EDP considers the relationship, no matter how distant, between the researcher and the tissue donor themselves and must clearly demarcate the responsibilities between clinical services and researchers. ${ }^{71}$ Although biobanks may act as custodians of the patient's biospecimens and researchers for the EDP, the clinicians are required to make decisions about patient care and the need to respond to any new information about the patient's disease generated by the research. Therefore, the risks inherent in the debate around return of results will not be clarified through EDPs, shifting responsibilities, and custodianship. Risk arises when no clear line of responsibility for the return of results is established.

4. Harmonization, standardization, certification, or accreditation: One of the risks of biobanking is driven by the concern over research reproducibility. ${ }^{72}$ This has placed emphasis on understanding the influence that tissue handling procedures have on the biological qualities of the biospecimens, ${ }^{73,74}$ bringing the discipline of "biospecimen research" into prominence. ${ }^{75}$ In order to manage this risk, biobanks are urged to follow common practice by entering networks, ${ }^{27}$ following standardized practice,,${ }^{9,76}$ harmonizing interoperability, ${ }^{77-79}$ and become certified ${ }^{80}$ or accredited. ${ }^{18}$ Biobanking initiatives are being introduced, which build standards and guidelines that seek, among others things, to boost cooperation and sharing of biospecimens between facilities. ${ }^{81}$ However, for top-down strategies like 
certification or accreditation from state-wide or national bodies to benefit biobanking, trust on the infrastructure is required. ${ }^{3}$ However, the requirements from “today's scientific output measurement strongly encourages individualism among scientists making cooperation and beneficial sample sharing difficult to establish." "19 This leads to the guiding concern in research-biobank relationships on the subject of ownership at the expense of building trust.

Consequence of message 3: Biobanking is a risky undertaking.

\section{Message 4: Biobanking requires unique funding models}

The true costs of developing and maintaining (biobank) operations, which may have a variety of funding sources, must be better understood. ${ }^{82}$

Although biobanks are important enablers of biomedical research, lead biobank professionals suggest that biobanks can be multimillion dollar operations, ${ }^{82}$ with each case collected by a biobank costing over US\$1000. ${ }^{83}$ Biobanks require organizations to invest in new technologies, orphan drug legislation, networking, researcher-community engagement, patient registries, objective assessments, bioinformatics support and databases, policy development and governance, along with ethical and legal frameworks. ${ }^{12}$ With this conflated expectation of biobank infrastructure, along with a continual focus on operational challenges, sustainability of funding has become a concern. ${ }^{17,38}$ "Even a modest biobank represents a significant funding commitment" 12 and finding financial support continues to be a major hurdle. ${ }^{84}$

Biobanks have followed different strategies in order to meet the funding shortfalls. As the typical research funding streams diminish, biobanks have recognized the need to "remodel" themselves. ${ }^{85}$

1. Cost recovery models: Often promoted as a preferred model, ${ }^{82}$ cost recovery to fund biobanks has been proven oversimplified and insufficient with at best $25 \%$ of cost being recovered ${ }^{83}$ and is generally out of favor.

2. Business models: Increasingly, biobank managers think in terms of business principles, market forces, price parity, inventory turnover, supply and demand, value chains, profitability, and returns. ${ }^{82}$ For example, the Beaumont Health System BioBank took a business model approach to consolidate its role within the hospitals by using CAP accreditation and Kaizen process improvement in order to gain confidence, productivity, and commercial sustainability. ${ }^{86}$ Within these considerations, there is an appreciation of competitiveness, bargaining power, profitability, customer satisfaction and marketing, ${ }^{87}$ concepts that conflict with the altruistic nature of tissue donation, and ideals of academia in health care. ${ }^{46}$

3. Biobank network models: In order to provide more sustainable funding models, major funders require biobanks to build networks. Government-based bodies, for example, the European Union, have created the Biobanking and BioMolecular resources Research Infrastructure and EuroBioBank, whereas the US National Institutes of Health supports Rare Disease Human Biorepositories and Biospecimens and the Office of Biorepositories and Biospecimen Research. ${ }^{12,13,78,88,89}$ In NSW, The Cancer Institute of NSW Translational Cancer Research Centre funding model supports only collaborative consortia, generally based around geographical locations. ${ }^{90}$

Although biobank sustainability is in question, costeffectiveness of biobanks is similarly under scrutiny. ${ }^{83}$ Likely these lines of enquiry are deeply related. Here is the conflicting message: the stated "importance" of biobanks to our translational research efforts is not reflected in their financial security. If they are so vital, why aren't they funded? For example, despite producing a major document in $2009^{26}$ guiding the national biobanking agenda, Australia's major funding body for medical research, the National Health Medical Research Council, decided to cease a major funding stream for biobank networks in 2013 because of the absence of a demonstrable "effect" on research deliverables. ${ }^{62}$

Consequence of message 4: Biobanking is not value for money.

\section{Message 5: Biobanking is an effective activity for medical research}

Biobanking has the potential to be the most powerful single platform for health innovation and knowledge generation provided that it is adequately resourced and networked. ${ }^{78}$

In 2009, Time magazine pronounced biobanks as one of the top 10 best innovations. ${ }^{1}$ Four years later, it was reported that biobanks are not reaching their potential. ${ }^{91}$ Indeed, only a few reports describe the extent of research supported by open access biobanks. ${ }^{13,92,93}$ In 2009, Australia's National Health and Medical Research Council ${ }^{26}$ drafted a major detailed discussion of all issues pertaining to biobanking with one notable exception. How do we determine whether a biobank is "effective"? The following questions should be asked: 
1. Has translational research been better off with biobanks? Hughes et $\mathrm{al}^{93}$ report that demand for samples has increased, yet over the same time period, the number of publications has not risen, suggesting that biobanks are providing researchers more samples per study. Text mining of cancer research publications verifies the increased use of human tissue samples in cancer research over the last two decades. ${ }^{94,95}$ However, these studies may not demonstrate the value in biobanking per se but rather demonstrate an investigator's ability to use tissues in research. Techniques that rapidly and routinely extract genomic and proteomic derivatives from the ever decreasing amounts of tissue have allowed researchers a means to use tissue more effectively in order to answer their questions. Use of these techniques is independent of collection strategies.

2. Do biobanks create more research opportunity? A US survey conveyed concerns that banked samples were being underutilized, ${ }^{38}$ while the Singapore BioBank was shut down because of lack of use. ${ }^{96} \mathrm{~A}$ Canadian study of health research infrastructure used in cancer studies reported that biospecimens contribute to $<40 \%$ of published cancer studies. Of most interest is that for studies using biospecimens only $31 \%$ were obtained from biobanks, whereas $46 \%$ were still obtained from pathology departments. ${ }^{97}$

3. Do biobanks accelerate scientific discovery? We are told that "the pre-eminent goal of biobanks is to accelerate scientific discovery and support improvements in healthcare through supply of high quality biospecimens." ${ }^{83}$ Many tissue banks do not focus on the utilization of their specimens in research, ${ }^{38,80,98}$ instead create biohoards where scientific discovery is not facilitated. ${ }^{31,99}$ Many biobanking initiatives are not transparent, having restricted access conditions, thus limiting their use in research. ${ }^{100}$ A litany of reasons for not sharing samples have been recorded: legal, ethical, intellectual property, commercialization, logistics, and sample quality. ${ }^{91}$ These trends have sparked international attempts to guide the harmonization of access conditions across biobanks through the development of charters. ${ }^{101}$

To date, the jury is still out on whether biobanking is truly effective in supporting translational research. Rather than accelerating research, it is likely that biobanking has increased the level of regulation that an investigator is required to negotiate, leading to inefficiency and unnecessary wastage. ${ }^{102}$

Consequence of message 5: Biobanking is inefficient.

\section{Getting back to basics}

Biobanking tries to present a positive message, which, in turn, has raised new and more complex underlying questions, many of which have not been satisfactorily addressed, conveying a more disparaging message of complexity, inefficiency, and risk. The message that we present is indeed a true reflection of the current state of biobanking practice. Therefore, we present a case that to negotiate these overly convoluted considerations and tangled opinions around biobanking, first we need to simplify our message. This in turn requires biobanks to consider some of the fundamental truths that are often forgotten, but that guide the use of human tissues in research applications.

\section{Fundamental I: It is about the verb not the noun}

We start with the premise that biobanking is more important than building biobanks. The activity of transferring biospecimens from donor to researcher is the fundamental purpose of a biobank. Biobankers should not be motivated to simply "store" as much tissue as they can, otherwise they will be guilty of biohoarding. ${ }^{31}$ Furthermore, a piece of tissue stored in a freezer is of little consequence. Only when we have strategies for transferring the biospecimens to scientists who draw out the information within that tissue sample that applies to human health, the significance of the biospecimens becomes apparent. This information must be revealed if the exercise of biobanking is to be of any value. Biobankers must be motivated by these broader research goals and must engage in the process of discovering new information regarding any human disease. Invariably, the issues related to biobanking have nothing to do with the operations of a biobank. Issues such as consent, return of results, clinical implementation, logistics, ethics, and legalities do not pertain to the systematic collection and processing of the tissue per se, but more to the reasons why we need to study the tissue in the first place. Becoming "tissue-handling experts" who know what researchers need to conduct robust investigations should be the biobankers purpose leading to the transfer of tissue specimens from donors to investigators and for which storage of the specimen for a period of time may be required.

With this fundamental principle, it is reasonable to suggest that spending time deliberating over an accepted definition of a "biobank" is facile. ${ }^{3}$ Indeed, the term "biobank" could cease being used altogether if it was accepted that the purpose of biobanking is at stake and that the operations of a specific facility is directed by that purpose. With this fundamental understanding, we will soon see that biobanking 
activities permeate throughout the clinical centers where routine and systematic tissue handling occurs throughout hospitals, pathology laboratories, surgeries, and clinics. These activities need to be monitored, not just those of an exclusive facility, creating systems and rules for all and avoiding exceptionalism. It is biobanking activity that is vital to medical research, not biobanks.

\section{Fundamental 2: Improved health care is utterly dependent on research}

Effective translation of [...] new knowledge, mechanisms and techniques generated by advances in basic science research into new approaches for prevention diagnosis and treatment of disease [...] is essential to improve human health. ${ }^{103}$

A fundamental truth that is often forgotten by modern health care centers is that every improvement in clinical practice and advance in patient outcomes has been driven by the work of researchers. Translational research is, and always will be, intimately linked with clinical care of patients. In order to understand human health, patients need to be studied. Consequently, the use of biospecimens and/or patient-specific data in scientific investigation is foundational to the translational research. In short, research using human biospecimens underpins improvements to patient health. Equally, biobanking is at the heart of translational research.

If policies are formed on the basis that all health care advancements have been built on the data generated from the investigations of the researcher, some of which involves biospecimens, it soon becomes apparent that clinical care and research investigations form a continuum of knowledge and are not mutually exclusive. Biobanking must be seen in view of this continuum. Can biobanking then be considered an exceptional activity separate from other tissue handling practices within the institutions? Likewise, as all biospecimens come from patients, and biomedical science is about determining what is wrong with these patients, does bioobjectification of the tissue samples yield the patient-centered focus that brings clinical relevance to translational research? The recent purchase by a UK biotech company of a collection of biospecimens from centenarian Sardinians brings the issue of commodification into prominence where the break in this clinico-research continuum for the sake of profit has left a community wondering how their donations will be of benefit to the wider population and themselves. ${ }^{46}$

Logically, it follows that how much we value translational research will be reflected in how institutions operate biobanks within their daily practices and where they place them within their organizational structures. As research involving human tissue becomes increasingly "personalised," 10 the distinction between the health provider and the researcher is blurring, and hospitals cannot ignore research findings but must establish procedures in order to implement the research findings into the clinic. Consequently, interventional centers (hospitals and clinics) must now have policies and practices in place to allow for research findings to be validated in individuals, clinical pathways to follow, and a clear mandate from the patients to pursue these findings on their behalf. Rather than biobanks being given special attention leading to organizational isolation, biobanking is integral to the outcomes of translational research, that is, improved health outcomes for patients.

\section{Fundamental 3: Biobanking is founded on relationships}

Biobanks as the well-guarded treasures in the interest of patients. $^{51}$

Biobanking always starts with a conversation with a donor, which commences a relationship. This fundamental act is at the core of all ongoing interactions with biobanking practice. Biobanking is promoted as an act of guardianship or stewardship over an individual patient's tissue samples. ${ }^{49}$ Hansson $^{61}$ poses that there is no need for a convoluted framework of rules and regulations around biobanking. Equally, Charo ${ }^{104}$ states that the debate around research using human tissues should be about identifying a proper balance between respect for patients and the collective interests of the research. ${ }^{104}$ As we are moving into an era of individualization of medical research, it is noteworthy that the community is being required to engage directly with the research agenda, assist prioritization of biospecimen usage, and being asked to ensure that the desires, needs, and values of the society are maintained. ${ }^{105-107}$ Our regulatory mechanisms must engender the continuance of patient dialogue with not only the clinician but also the biobankers and researchers. ${ }^{33}$

Biobanking introduces the general public into the world of research. The messages from biobanks to donors and the public are therefore crucial. However, the general public are educated neither about biobanking nor the value of research using biospecimens. ${ }^{105}$ Hence, how we value a piece of tissue and its role in research will influence the message presented to the public. If we present tissues based on Thompson's 'rubbish theory' 108 where biobanks are in the business of revalorization of "left over" tissue, ${ }^{53}$ then we draw on the altruistic nature of the donor. However, if we present that 
the tissue value is in its ability to inform us about the individual patient, we draw upon the self-determining needs of the patient donor. Minority cultural groups are guarded with respect to their involvement in biomedical research and biobanking because of the absence of beneficial relevance and potential for harm, mostly cultural stigmatization, of the research to those communities. ${ }^{46,105,109}$ The emergence of the "biorights" movement has challenged the assumption of altruism in tissue donation. ${ }^{110}$ In return for use of their tissue and access to clinical data, donors seek financial compensation, return of medical information, influence over how samples are studied, and the questions pursued as well as a stake in any commercial benefit gleaned from the research. Such imposing requirement from the public on researchers has been met with angst. The conversations with donors may become more of a negotiation resulting in research efforts being bogged down. This new state of affairs further highlights how biobanking activity has opened up the need for increased engagement with the communities we seek to serve through translational research highlighting the need to create trust between researchers and donors. ${ }^{96,111,112}$

If focus on the ethics around the use of tissue in research heightens the fear that biobanking is risky, then returning to this fundamental principle and building trust in the relationships should engender public confidence in what is being done.

\section{Fundamental 4: Hospitals are biobanks}

Embedding biobank within a healthcare setting has the potential to address many of the challenges that had been identified in the translational research setting. ${ }^{113}$

It is a foundational truth that, regardless of the biobanking model, the vast majority of human tissues that end up in biobanks are collected at hospitals through interaction with clinicians, surgeons, and pathologists. ${ }^{7}$ The Australian Breast Cancer Tumour Bank recognized that engagement from local medical and clinical services to help champion the collection of biospecimens is vital. ${ }^{30}$ With the growth of biobanking, it has been realized that "pathology is the cornerstone of hospital-based tissue biobanking." 114 Involvement of pathologists has been recognized as central to biobanking, adding scientific value, clinical assessment, real-world application, and intellectual property. ${ }^{114}$ Current assessment of research publications indicates that cancer research using biospecimens has indeed been supported more by pathology departments than biobanks. ${ }^{97}$ Indeed, if we consider the pathology departments of hospitals as "biobanks," materials from over 30,000 breast cancer patients could be made available across Europe. ${ }^{99}$

Despite the push for biobank exceptionalism, biobanks and pathology departments of hospitals must operate in lockstep with each other with respect to the same ethical principles, legal limitations, patient privacy, quality assurance, fit-for-purpose standards, and data linkage requirements. ${ }^{5,71}$ Newborn screening collections, from the viewpoint of being a "biobank," highlight how regulation of informed consent will start to impose on their practice, recognizing that they can be used for research in the same way as biobanks. ${ }^{32}$ The Sample PREanalytical Code, ${ }^{76}$ used by biobanks, is none too dissimilar to the standards being introduced into pathology in preparation for personalized genomics, ${ }^{115}$ highlighting how tissue handling and data reproducibility are becoming vital to understand human disease and its treatment by all departments.

Biobanks are therefore a public health issue. Consistent attempts to partition clinical management from research infrastructure impede the progress in health management and improved outcomes ${ }^{116}$ leading to the recommendation that professional biobanks become "integrative service infrastructure" in hospitals. ${ }^{6,9,19,117}$ Two of the primary activities undertaken by hospitals for the clinical care of patients are the collection of tissue biopsies, including their long-term storage or archiving and the linkage to personal clinical and demographic data. This is done with the goal of performing laboratory tests with data correlations in order to characterize the illness in patients. Indeed, these goals are identical to those of biobanks; the collection of biospecimens is linked to personal clinical data in order to facilitate research investigations that will allow experts to characterize the patient. The primary difference between biobanks and pathology tissue handling is what can be done with the information generated especially in the context of the clinical care of patients.

It has been pointed out that "the greatest barrier to research biopsies is not patient reluctance, but rather that of the physician." ${ }^{17}$ As hospitals are the recipients of the information derived from investigations in human tissues, which may direct clinical intervention for patients, there can be no more passive acceptance of research by hospital management! Indeed, I would propose that how closely located and operationally embedded biobank infrastructure is within the pathology and clinical departments of a hospital, reflects how the health professionals value the latest health and biomedical research findings. Even non-hospital-based biobanks have come to the realization that they are best operated as close to the source of the biospecimens, the patients. ${ }^{118}$ 
It is noteworthy that biobanking may become less of a fiscal burden if it was undertaken within the clinical and pathology settings as part of the ongoing practices already performed within hospitals. Remodeling biobanks may indeed result in the return to their foundations purely to become economically viable.

\section{Fundamental 5: Biospecimens are "packages of information"}

One could go further and ask whether the current biomedical ethics review system is inadequate - indeed inappropriate in these "data-driven research" contexts. ${ }^{119}$

The fundamental truth that biobanking is about gaining knowledge is not in question. However, references to the generation of "information" and "data" from biospecimens are often posed as a possible consequence of biobanking. NSW Health policy dictates that "prospective participants should be informed that, while research utilising biobank specimens is not designed to improve the health of the individual participant, discoveries may be made during the course of the research, and serious and significant findings returned to them. ${ }^{28}$ In truth, in this situation, that the samples were biobanked is inconsequential to whether the biospecimens yield information in the laboratory nor whether that information may be required to be returned to the participant. Biospecimens obtained from pathology departments, doctors, surgeries, and clinical trials all have the potential of discovering new information pertaining to individual patients, which is the requirement in health care! What matters is whether the information generated by the scientific process provides insight that is useful for the patient. ${ }^{41}$

A researcher's interest in a biospecimen is not because it is a "biospecimen," but rather because it comprises information needed to understand the biological causes and mechanism of a disease. Biospecimen value is created by annotation, analysis, or transformation of specimens to other derivatives. ${ }^{16}$ The growth of genomics has highlighted that biospecimens are purely a source of information about patients, their predispositions and genetic heritage. This information carries greater ethical weight due to the potential impact this may have on individuals, their families, and their community. The management of human tissue in research therefore inevitably leads to a requirement to manage the data generated from laboratory investigations which may be linked to clinical metadata. As has been highlighted by Louk, ${ }^{41}$ with biobanking, "the main harm is informational," not physical or psychological. This raises the "bio-virtual" concept where a form of life exists as information, data, or informational flow that mobilizes the bio-object, making it more purposeful. ${ }^{42}$

This makes biobanking fundamentally an informatics problem. For example, with respect to biospecimen science, the solutions to tissue quality do not come down to developing highly specific tissue handling steps, many of which would be unrealistic in a busy hospital setting. Rather, the solution is to note down specific parameters, capturing the provenance of the biospecimens and then determining through multivariate statistics whether these handling conditions become influencing covariates. ${ }^{75}$ Furthermore, ethics considerations are turning from deliberations on how tissues are obtained to how we manage the deluge of personal genetic information that is rapidly extracted from the biospecimens. ${ }^{119}$ Biobanks and bioinformatics are inextricably linked. This highlights that if biospecimens are considered primarily as "sources of information," then they should be considered within an ethical framework that addresses personal information generally and how this information is managed ethically in the patient context.

Exploration around the establishment of disease registries may be informative. At the most fundamental level, registries collect data about patients and improve our knowledge of human disease. ${ }^{88,120}$ If, indeed, biospecimens are considered sources of information about patients and their diseases, then a biobank can be considered a "registry." Most importantly, biobanks perform the task of linking the biological information derived from the biospecimen to the clinical, phenotype, and response data derived from the patients' journey through the medical system. As disease registries are databases, many of the issues faced are based on information technology, with naming conventions, interoperability, security, accessibility, and sustainability as key concerns. Biobanks need to address all these issues. It has been contended for disease registries that "the quality of the system in which the data is contained becomes a significant bottleneck." ${ }^{120}$ So it is with the biobanks.

By focusing on the biospecimen collection and distribution practices, biobanks will be gauged by supply and demand principles and judged on the efficiency of their ability to move a "product." However, by accepting the fundamental truth that biospecimens are sources of information, biobanks should be judged on their ability to generate knowledge, discovery of clinical innovations, and implementation of new practices that lead to improved health outcomes for patients.

\section{Disclosure}

The author reports no conflicts of interest in this work. 


\section{References}

1. Park A. 10 Ideas changing the world right now. Time Magazine, 2009. Available from: http://content.time.com/time/specials/packages/article/0,28804,1884779_1884782_1884766,00.html. Accessed November 2, 2016.

2. Vaught J, Kelly A, Hewitt R. A review of international biobanks and networks: Success factor and key benchmarks. Biopreserv Biobank. 2010;7:143-150.

3. Beier K, Lenk C. Biobanking strategies and regulative approaches in the EU: recent perspectives. J Bioreposit Sci Appl Med. 2015; 3:69-81.

4. De Souza YG, Greenspan JS. Biobanking past, present and future: responsibilities and benefits. AIDS. 2013;27:303-312.

5. Chadwick D, Roehrl MHA. High-quality biobanking for personalised precision medicine: BioSpecimens at the helm. Diagn Histopathol. 2013;19:447-455.

6. Pelagio G, Pistillo D, Mottolese M. Minimum biobanking requirements: issues in a comprehensive cancer centre biobank. Biopreserv Biobank. 2011;9:141-148.

7. Grizzle WE, Aamodt R, Clausen K, et al. Providing human tissue for research. Arch Pathol Lab Methods. 1998;122:1065-1076.

8. Esgueva R, Park K, Kim R, et al. Next-generation prostate cancer biobanking: toward a processing protocol amenable for the International Cancer Genome Consortium. Diagn Mol Pathol. 2012;21: 61-68.

9. Mee B, Gaffney E, Glynn SA, et al. Development and progress of Ireland's Biobank Network: ethical, legal, and social implications (ELSI), standardized documentation, sample and data release, and international perspective. Biopreserv Biobank. 2013;11:3-11.

10. Hewitt RE. Biobanking: the foundation of personalized medicine Curr Opin Oncol. 2010;23:112-119.

11. Lochmüller H, Aymé S, Pampinella F, et al. The role of biobanking in rare diseases: European Consensus Expert Group report. Biopreserv Biobank. 2010;7:155-156.

12. Graham CE, Molster C, Baynam GS, et al. Current trends in biobanking for rare diseases: a review. J Bioreposit Sci Appl Med. 2014; 2:49-61.

13. Mora M, Angelini C, Bignami F, et al. The EuroBioBank Network: 10 years of hands-on experience of collaborative, transnational biobanking for rare diseases. Eur J Hum Genet. 2014;272:1-8.

14. Riegman PHJ, van Veen E-B. Biobanking residual tissues. Hum Genet. 2011;130:357-368.

15. Brisson AR, Matsui D, Rieder MJ, et al. Translational research in pediatrics: tissue sampling and biobanking. Pediatrics. 2011;129: 153-162.

16. Zatloukai K, Hainaut P. Human tissue biobanks as instruments for drug discovery and development: impact on personalized medicine. Future Med. 2010;4:895-903.

17. Basik M, Aguilar-Mahecha A, Rouseau C, et al. Biopsies: next generation biospecimens for tailoring therapy. Nat Rev Clin Oncol. 2013;10: 437-450.

18. Herpel E, Rocken C, Manke H, Schirmacher P, Flechtenmacher C. Quality management and accreditation of research tissue banks: experience of the National Center for Tumour Diseases (NCT) Heidelberg. Virchows Arch. 2010;457:741-747.

19. Riegman PHJ, de Jong BWD, Llombart-Bosch A. The Organisation of European Cancer Institute Pathobiology Working Group and its support of European biobanking infrastructure for translational cancer research. Cancer Epidemiol Biomarkers Prev. 2010;19:923-926.

20. Vegvari A, Welinder C, Lindberg H, Fehniger TE, Marko-Varga G. Biobank resources for future patient care: developments, principles and concepts. J Clin Bioinforma. 2011;1:24.

21. Goldblatt EM, Lee W-H. From bench to bedside: the growing use of translational research in cancer medicine. Am J Transl Res. 2010; $2: 1-18$

22. Dancey J. Genomics, personalized medicine and cancer practice. Clin Biochem. 2012;45:379-381.
23. McKeon S. McKeon review: we need to integrate research and health services. Transforming the Nation's Healthcare website. 2012 October. Available from: http://www.transformingthenation.com.au/2012/10/ mckeon-review-we-need-to-integrate-research-and-health-services/. Accessed November 2, 2016.

24. NSW government response to the NSW health and medical research strategic review. NSW Government Ministry of Health website; 2012. Available from: http://www.health.nsw.gov.au/ohmr/Publications/ stratreview-gov-response.pdf. Accessed November 2, 2016.

25. Boyer GJ, Whipple W, Cadigan RJ, Henderson GE. Biobanks in the United States: how to identify an undefined and rapidly evolving population. Biopreserv Biobank. 2012;10:511-517.

26. National Health and Medical Research Council, Biobank Information Paper, 2010. Available from: https:/www.nhmrc.gov.au/guidelinespublications/e110. Accessed November 2, 2016.

27. Hewitt R, Hainaut P. Biobank is a fast moving world: an international perspective. J Natl Cancer Inst Monogr. 2011;42:50-51.

28. Consistent State-wide Consent for Research Biobanking Consultation Paper. NSW Health Pathology website. 2015 March. Available from http://www.health.nsw.gov.au/ohmr/biobankregistry/Documents/ biobank-consent-public-consultation.pdf. Accessed November 2, 2016.

29. Hewitt R, Watson P. Defining biobank. Biopreserv Biobank. 2011; 11:309-315.

30. Carpenter J, Clarke CL. Biobanking sustainability - Experiences of the Australian Breast Cancer Tissue Bank (ABCTB). Biopreserv Biobank 2014;12:395-401.

31. Catchpoole DR. Biohoarding: treasures not seen, stories not told $J$ Health Serv Res Policy. 2015;21:140-142.

32. Knoppers BM, Avard D, Senecal K. Newborn screening programmes: emerging biobanks? Norsk Epidemiologi. 2012;21:163-168.

33. Cambon-Thomsen A, Rial-Sebbag E, Knoppers BM. Trends in ethical and legal frameworks for the use of human biobanks. Eur Respir J. 2007;30:373-382.

34. Kaye J. Do we need uniform regulatory system for biobanks across Europe. Eur J Hum Genet. 2006;14:245-248.

35. Shaw DM, Elger BS, Colledge F. What is a biobank? Differing definitions among biobank stakeholders. Clin Genet. 2014;85:223-227.

36. Morente MM, Fernández PL, de Alava E. Biobanking: old activity or young discipline. Semin Diagn Pathol. 2008;25: 317-322.

37. Strong DM. The US Navy Tissue Bank: 50 years on the cutting edge. Cell Tissue Bank. 2000;1:9-16.

38. Henderson GE, Cadigan RJ, Edwards TP, et al. Characterizing biobank organizations in the US: results from a national survey. Genome Med. 2013;5:3

39. A comprehensive review of cancer-related biobanks in New South Wales, Oct 2009. Cancer Institute NSW Monograph. Available from: https://www.cancerinstitute.org.au/how-we-help/reports-andpublications/a-comprehensive-review-of-cancer-related-biobanks. Accessed November 2, 2016

40. Rush A, Christiansen JH, Farrell JP, et al. Biobank classification in an Australian setting. Biopreserv Biobank. 2015;13:212-218.

41. Louk K. Rethinking the ethics of human biomedical non-interventional research. In: Schildmann J, Sandow V, Rauprich O, Vollmann J, editors. Human Medical Research. Basel: Springer; 2012: Chapter 15, $175-184$

42. Holmberg T, Schwennesen N, Webster A. Bio-objects and the bioobjectification process. Croatian Med J. 2011;52:240-242.

43. Tupasela A, Stephens N. The boom and bust cycle of biobanking - thinking through the life cycle of biobanks. Croatian Med J. 2013;54:501-503.

44. Stephens N, Dimond R. Unexpected tissue and the biobank that closed an exploration of value and the momentariness of bio-objectification processes, Life Sci Soc Policy. 2015;11:14.

45. Boeckhout M, Douglas CMW. Governing the research-care divide in clinical biobanking: Dutch perspective. Life Sci Soc Policy. 2015;11:7.

46. Kirchgaessner S. Ethical questions raised in search for Sardinian centenarians' secret. The Guardian. 2016 Aug 13. Available from: https:// www.theguardian.com/world/2016/aug/12/ethical-questions-raised-insearch-for-sardinian-centenarians-secrets. Accessed November 2, 2016 
47. Gibbons SMC, Kaye J. Governing genetic databases: collection, storage and use. Kings Law J. 2007;18:201-208.

48. Knoppers BM, Hudson TJ. The art and science of biobanking. Hum Genet. 2011;130:329-332.

49. Henderson GE, Edwards TP, Cadigan RJ, et al. Stewardship practices of U.S. biobanks. Sci Transl Med. 2013;5:1-6.

50. Brenner J. Establish a transparent chain-of-custody to mitigate risk and ensure quality of specialized samples. Biopreserv Biobank. 2010;7:151-153.

51. Oosterhuis JW, Coebergh J, van Veen EB. Tumour banks, well-guarded treasures in the interest of patients. Nat Rev Cancer. 2003;3:73-77.

52. Hansson MG. Ethics and biobanks. Br J Cancer. 2009;100:8-12.

53. Morrell B, Lipworth W, Axler R, Kerridge I, Little M. Cancer as rubbish: donation of tumour tissue for research. Qual Health Res. 2011; 21:75-81.

54. Pillai U, Phillips K, Wilkins G, et al. Factors that may influence the willingness of cancer patients to consent for biobanking. Biopreserv Biobank. 2014;12:409-413.

55. Mancini J, Pellegrini I, Viret F, et al. Consent for biobanking: assessing understanding and views of cancer patients. J Natl Cancer Inst. 2011;103:154-157.

56. Porteri C, Pasqualetti P, Togni E, Parker M. Public's attitudes on participation in a biobank for research: an Italian survey. BMC Med Ethics. 2014;15:81.

57. Wendler D. One-time general consent for research on biological samples. Br Med J. 2006;332:544-547.

58. Coebergh JWW, van Veen E-B, Vandenbroucke JP, van Diest P, Oosterhuis W. Opt out system for patient is optimal and endorsed in many countries. BMJ. 2006;332:665.

59. Johnsson L, Hansson MG, Eriksson S, Helgesson G. Patients' refusal to consent to storage and use of samples in Swedish biobanks: cross sectional study. BMJ. 2008;337:224-226.

60. Laurie G. Consent for biobanking: lack of dissent when opting in doesn't necessarily support 'opt out'. BMJ. 2008;337:186-187.

61. Hansson MG. The need to downregulate: a minimal ethics framework for biobank research. Methods Mol Biol. 2011;675:39-59.

62. Gedye C, Fleming J. Forsaking cures for cancer: why are we discarding the tumour biospecimens of most patients? Med J Aust. 2016; 204:297-298.

63. Holm S. Informed consent and the bio-banking of material from children. Genom Soc Policy. 2005;1:16-26.

64. Hens K, Cassiman J-J, Nys H, Diericks K. Children, biobanks and the scope of parental consent. Eur J Hum Genet. 2011;19:735-739.

65. Rush A, Battisti R, Barton B, Catchpoole D. Opinions of young adults on re-consenting for biobanking. J Paediatrics. 2015;167:925-930.

66. Goldenberg AJ, Hull SC, Botkin JR, Wilfond BS. Pediatric biobanks: approaching informed consent for continuing research after children grow up. J Pediatrics. 2009;155:578-583.

67. Offit K. Personalized medicine: new genomics, old lessons. Hum Genet. 2011;130:3-14.

68. Knoppers BM, Avard D, Senecal K, Zawati MH along with the PG International Paediatrics Platform members. Return of whole-genome sequencing results in paediatric research: a statement of the P3G international paediatric platform. Eur J Hum Genet. 2014;22:3-5.

69. Bredenoord AL, van Delden JJM. Research ethics in genomic research: feedback of individual genetic data to research participants. In: Schildmann J, Sandow V, Rauprich O, Vollmann J, Editors. Human Medical Research. Basil: Springer: 2012:127-135.

70. National Statement on Ethical Conduct in Human Research 2007. National Health and Medical Research Council. Updated May 2015. Available from: https://www.nhmrc.gov.au/_files_nhmrc/publications/ attachments/e72_national_statement_may_2015_150514_a.pdf. Accessed November 2, 2016.

71. Zeps N, Bledsoe MJ, Managing the ethical issues of genomic research using pathology specimens. Clin Biochem Rev. 2015;36:21-27.

72. Rush A, Spring K, Byrne JA. A critical review of cancer biobank practice in relation to biospecimen quality. Biophys Rev. 2015;7:369-378.
73. Enroth S, Hallmans G, Grankvist K, Gyllensten U. Effects of long-term storage time and original sampling month on biobank plasma protein concentration. EBioMedicine. 2016;12:309-314.

74. Jones RJ, Boyce T, Fennell M, et al. The impact of delay in cryo-fixation on biomarkers of Src tyrosine kinase activity in human breast and bladder cancers. Cancer Chemother Pharmacol. 2008;61:23-32.

75. Betsou F, Barnes R, Burke T, et al. Human biospecimens research: Experimental protocol and quality control tools. Cancer Epidemiol Biomarkers Prev. 2009;18:1017-1025.

76. Betsou F, Lehmann S, Ashton G, et al. Standard preanalytical coding for biospecimens: defining the sample PREanalytical Code. Cancer Epidemiol Biomarkers Prev. 2010;19:1004-1011.

77. Lee D, Lee Y. The Korean National Research Resource Centre. Biopreserv Biobank. 2010;7:137-142.

78. Harris JR, Burton P, Knoppers BM, et al. Toward a roadmap in global biobanking for health. Eur J Hum Genet. 2012;20:1105-1111.

79. Cervo S, De Paoli P, Perin T, Canzonieri V, Steffan A. Cost-effective organization of an institutional human cancer biobank in a clinical setting: CRO-Biobank experience toward harmonization. Int J Biol Markers. 2015;30:e243-e251.

80. Grizzle WE, Guner EW, Sexton KC, Bell WC. Quality management of biorepositories. Biopreserv Biobank. 2015;13:193-194.

81. Riegman PHJ, de Jong B, Daidone MG, et al. Optimizing sharing of hospital biobank samples. Sci Transl Med. 2015;7:297.

82. Vaught J, Rogers J, Carolin T, Compton C. Biobankonomics: developing a sustainable business model approach for the formation of a human tissue biobank. J Natl Cancer Inst Monogr. 2011;2011:24-31.

83. Albert M, Bartlett J, Johnston RN, Schacter B, Watson P. Biobank bootstrapping: Is biobank sustainability possible through cost recovery? Biopreserv Biobank. 2014;12:374-380.

84. Chandras C, Weaver T, Zouberakis M, et al. Models for financial sustainability of biological databases and resources. Database. 2009; bap017:1-9.

85. Parry-Jones A. Assessing the financial, operational and social sustainability of a biobank: The Wales Cancer Bank case study. Biopreserv Biobank. 2014;12:381-388.

86. Wilson GD, D'Angelo K, Pruetz BL, Geddes TJ, Larson DM, Akervall J. The challenge of sustaining a hospital-based biobank and core molecular laboratory: The Beaumont Experience. Biopreserv Biobank. 2014;12:306-311.

87. Warth R, Perren A. Construction of a business model to assure financial sustainability of biobanks. Biopreserv Biobank. 2014;12:389-394.

88. Rubinstein YR, Croft SC, Bartek R, et al. Creating a global rare disease patient registry linked to a rare disease biorepository database: Rare Disease-HUB (RD-HUB). Contemp Clin Trials. 2010;31:394- 404.

89. Roazzi P, Benedetto CD, Bravo E, D'Agnolo G. Biobank networking: The European Network Initiative and the Italian Participation. Biopreserv Biobank. 2011;9:175-179.

90. Translational Cancer Research Centres NSW, Cancer Institute of NSW Website. Published Sept 2016. Available from: https://www. cancerinstitute.org.au/data-research/translating-research-into-practice/ funded-centres. Accessed November 2, 2016.

91. Colledge F, Elger B, Howard HC. A review of the barriers to sharing in biobanking. Biopreserv Biobank. 2013;11:339-346.

92. Zhou L, Catchpoole DR, Spanning the genomics era: the vital role of a single institution biorepository for rare disease research over a decade. Transl Paediatrics. 2015;4:93-106.

93. Hughes SE, Barnes RO, Watson PE. Biospecimen use in cancer research over two decades. Biopreserv Biobank. 2010;8:89-97.

94. Cole A, Cheah S, Dee S, et al. Biospecimen use correlates with emerging techniques in cancer research: impact on planning future biobanks. Biopreserv Biobank. 2012;10:518-525.

95. Braun L, Lesperance M, Mes-Massons A-M, Tsao MS, Watson PH. Individual investigator profiles of biospecimen use in cancer research. Biopreserv Biobank. 2014;12:192-198.

96. Wai CT. The closure of the national bio-bank in Singapore. Asia Pacific Biotech. 2012;16(4):40-44. 
97. Castillo_Pelayo T, Babinszky S, LeBlanc J, Watson PH. The importance of biobanking in cancer research. Biopreserv Biobank. 2015; 13:172-177.

98. Cadigan RJ, Lassiter D, Haldeman K, Conlon I, Reavely E, Henderson GE. Neglected ethical issues in biobank management: results from a U.S. study. Life Sci Soc Policy. 2013;5:3.

99. McIntyre P. Unlocking progress: why we need to change the culture of biobanking. Cancer World. 2016;72:46-50.

100. Verlinden M, Nys H, Ectors N, Huys I. Access to biobanks: harmonization across biobank initiatives. Biopreserv Biobank. 2014;12:422.

101. Mascalzoni D, Dove ES, Rubinstein Y, et al. International charter of principles for sharing bio-specimens and data. Eur J Hum Genet. 2015;23:721-728.

102. Salman RA, Beller E, Kagan J, et al. Increasing value and reducing waste in biomedical research regulation and management. Lancet. 2014;383:176-185

103. Woolf SH. The meaning of translational research and why it matters. JAMA. 2011;299:211-213.

104. Charo A. Body or research - ownership and use of human tissue. N Engl J Med. 2006;355:1517-1519.

105. Taualii M, Davis EL, Braun KL, et al. Native Hawaiian views on biobanking. J Cancer Educ. 2014;29:570-576.

106. Terzic A, Waldman SA. Translational medicine: path to personalized and public health. Biomark Med. 2010;4:787-790.

107. Steinsbekk KS, Solberg B. Biobanks - when is re-consent necessary? Public Health Ethics. 2011;4:236-250.

108. Thompson M. Rubbish Theory. Oxford, UK: Oxford University Press; 1979.

109. Haring RC, Henry WA, Hudson M, Rodriquez EM, Taualii M. Views on clinical trial recruitment, biospecimen collection, and cancer research: population science from landscapes of the Haudenosaunee (People of the Longhouse). J Cancer Educ. 2016 Epub July 9.
110. Daley B, Cranley E. 'Biorights' rise: donors demand control of their samples. SciPol Website; October 2016. Available from: http://sciencepolicy.duke.edu/content $/ \%$ E2\%80\%98biorights $\%$ E2 $\% 80 \% 99$-risedonors-demand-control-their-samples. Accessed November 2, 2016.

111. Hansson MG. Building on relationships of trust in biobank research J Med Ethics. 2005;31:415-418.

112. Boeckhout M, Reuzel R, Zielhuis G. The donor as partner: How to involve patients and the public in the governance of biobanks and registries. BBMRI Biobank Services Netherland; 2014. Available from: www.bbmri.nl/wp-content/uploads/2015/10/guidelineeng_def.pdf. Accessed November 2, 2016.

113. Kaye J. Embedding biobanks as tools for personalised medicine. Norsk Epidemiologi. 2012;21:169-175.

114. Bevilacqua G, Bosman F, Dassesse T, et al. The role of the pathologist in tissue banking: European Consensus Expert Group Report. Virchows Arch. 2010;456:449-454.

115. Groenen PJTA, Blokx WAM, Diepenbroek C, et al. Preparing pathology for personalized medicine: possibilities for improvement of the pre-analytical phase. Histopathology. 2011;59:1-7.

116. Reed W, Jor S, Brugn R. How can clinical biobanks and patient information be adapted for research - establishing a hospital based data warehouse solution. Norsk Epidemiologi. 2012;21:191-194.

117. Riegman PHJ, Dinjens WNM, Oosterhuis KJ. Biobanking for interdisciplinary clinical research. Pathobiology. 2007;74:239-244.

118. Cortés B, Schiffman M, Herrero R, et al. Establishment and operation of a biorepository for molecular epidemiologic studies in Costa Rica. Cancer Epidemiol Biomarkers Prev. 2010;19:916-922.

119. Knoppers BM. Consent to 'personal' genomics and privacy. $E M B O$ Rep. 2010;11:416-419.

120. Bellgard M, Beroud C, Parkinson K, et al. Dispelling myths about rare disease registry system development. Source Code Biol Med. 2013;8:21.
Journal of Biorepository Science for Applied Medicine

\section{Publish your work in this journal}

The Journal of Biorepository Science for Applied Medicine is an international, peerreviewed, open access journal that focuses on new developments and advances in the emerging and evolving field of biorepository science. This includes biospecimen procurement, processing, preservation, and banking for application to applied medicine. The Journal invites submission of manuscripts which address these aspects in addition to systems logic, clinical throughput and ethical issues pertaining to application of

\section{Dovepress}

biorepositories and their effects on clinical medicine. The journal is characterized by the rapid reporting of reviews, original research, methodologies, technologies and analytics in this subject area. The manuscript management system is completely online and includes a very quick and fair peer-review system, which is all easy to use. Visit http:// www.dovepress.com/testimonials.php to read real quotes from published authors.

Submit your manuscript here: https://www.dovepress.com/journal-of-biorepository-science-for-applied-medicine-journa| 